\title{
Weed impairs teen cognition
}

Am. J. Psychiatry https://doi.org/gd89cq (2018)

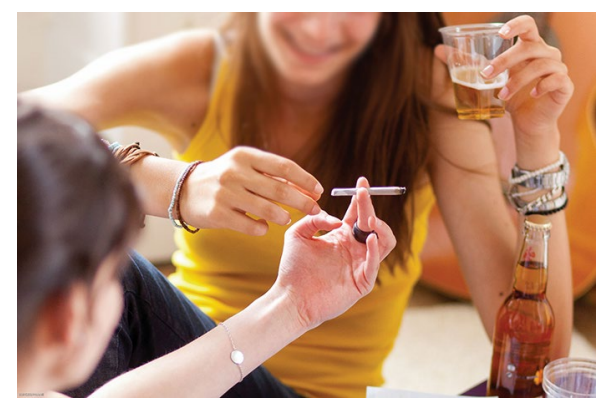

Credit: BURGER/Canopy/Getty

Alcohol and cannabis use during adolescence has been associated with impairments in learning, memory and attention, but it is not clear whether these cognitive impairments are a direct consequence of alcohol and cannabis use or instead represent an underlying vulnerability to substance misuse.

Patricia Conrod and her student, Jean-François Morin, of the University of Montreal, and colleagues set out to answer this question by measuring alcohol and cannabis use and cognitive abilities every year for four years in a population-based sample of 3,826 seventh-grade students. They find that individuals who were more likely to use either cannabis or alcohol had lower inhibitory control, perceptual reasoning and working memory, suggesting that these individuals are vulnerable to substance misue generally. However, they also found that cannabis use - and not alcohol use - was associated with impairments in working memory, recalling memories and perceptual reasoning. Cannabis use also had lasting effects: cannabis use in any given year was associated with impaired working memory and inhibitory control one year later.

Taken together, these results show cannabis use during adolescence is associated with observable delays in teen cognitive development, which were not detected for alcohol, and that the effects of cannabis on cognitive abilities can last.

Mary Elizabeth Sutherland

Published online: 8 November 2018 https://doi.org/10.1038/s41562-018-0482-5 